\title{
The Factorization of a Matrix as the Commutator of Two Matrices
}

\author{
John M. Smith* \\ Institute for Computer Sciences and Technology, National Bureau of Standards, \\ Washington, D.C. 20234
}

(April 4, 1974)

\begin{abstract}
Let $P=I_{p} \dot{+}\left(-I_{q}\right)$, the direct sum of the $p \times p$ identity matrix and the negative of the $q \times q$ identity matrix. The following theorem is proved.

Theorem: If $\mathrm{X}=\mathrm{cZ}$ where $\mathrm{Z}$ is a $4 \times 4 \mathrm{P}$-orthogonal, $\mathrm{P}$-skew-symmetric matrix and $|\mathrm{c}| \leqslant 2$, there exist matrices $\mathrm{A}$ and $\mathrm{B}$, both of which are $\mathrm{P}$-orthogonal and $\mathrm{P}$-skew-symmetric, such that $\mathrm{X}=\mathrm{AB}-\mathrm{BA}$. Methods for obtaining certain matrices which satisfy $X=A B-B A$ are given. Methods are also given for determining pairs of anticommuting $P$-orthogonal, $P$-skew-symmetric matrices.
\end{abstract}

Key words: Anticommuting; commutator; factorization; matrix; orthogonal; skew-symmetric.

\section{Introduction}

Let $P=I_{p} \dot{+}\left(-I_{q}\right)$, the direct sum of the $p \times p$ identity matrix and the negative of the $q \times q$ identity matrix. Katz and Olkin $[2]^{1}$ define a real matrix $A$ to be orthogonal with respect to $P(P$ orthogonal) if and only if

$$
A P A^{\prime}=P
$$

where $A^{\prime}$ is the transpose of $A$. Furthermore, they define $B$ to be skew-symmetric with respect to $P(P$-skew-symmetric $)$ if and only if $B P$ is skew-symmetric in the ordinary sense.

The main result of this paper is concerned with matrices which are both $P$-orthogonal and $P$-skew-symmetric of order $n=4=p+q$. Smith [7] proved that such matrices exist in only two cases, $p=4, q=0$ and $p=q=2$. In the first case $P$-orthogonal and $P$-skew-symmetric reduce to orthogonal and skew-symmetric in the ordinary sense.

Pearl [4] and Smith [6] proved the following theorem in the cases $p=4, q=0$ and $p=q=2$ respectively.

Theorem $\mathrm{J}$ : If the $4 \times 4$ matrices $\mathrm{A}$ and $\mathrm{B}$ are both $\mathrm{P}$-orthogonal and $\mathrm{P}$-skew-symmetric then their commutat . , $[\mathrm{A}, \mathrm{B}] \equiv \mathrm{AB}-\mathrm{BA}$, is a scaler multiple of a $4 \times 4$ P-orthogonal, $\mathrm{P}$-skew-symmetric matrix.

The purpose of this paper is to prove a converse to Theorem 1. Shoda [5] proved that if $X$ is a square matrix with zero trace having elements in an algebraically closed field then there exist matrices $A$ and $B$ such that $X=A B-B A$. Albert and Muckenhoupt [1] removed the restriction that the field be algebraically closed. However, both the method of Shoda and the method of Albert and Muckenhoupt give a singular matrix $B$. The main result of this paper is:

Theorem 2: If $\mathrm{X}=\mathrm{cZ}$ where $\mathrm{Z}$ is a $4 \times 4$ P-orthogonal, $\mathrm{P}$-skew-symmetric matrix and $|\mathrm{c}| \leqslant 2$, there exist matrices $\mathrm{A}$ and $\mathrm{B}$, both of which are $\mathrm{P}$-orthogonal and $\mathrm{P}$-skew-symmetric, such that $\mathrm{X}=\mathrm{AB}-\mathrm{BA}$.

AMS Subject Classification: Primary 15A21, Secondary 15A27.

*Also with George Mason University, Fairfax, Virginia '22030.

' Figures in brackets indicate the literature references at the end of this paper. 
Methods for obtaining certain matrices which satisfy $X=A B-B A$ are given. Methods are also given for determining pairs of anticommuting $P$-orthogonal, $P$-skew-symmetric matrices.

\section{Anticommuting Matrices}

In examining the structure of $P$-orthogonal, $P$-skew-symmetric matrices in the case $p=4$, $q=0$, Pearl [4] shows that any such matrix has exactly one of the following forms:

$$
\begin{array}{ll}
\text { (i) } \alpha_{1} R_{1}+\alpha_{2} R_{2}+\alpha_{3} R_{3}, & \alpha_{1}^{2}+\alpha_{2}^{2}+\alpha_{3}^{2}=1 \\
\text { (ii) } \alpha_{1} S_{1}+\alpha_{2} S_{2}+\alpha_{3} S_{3}, & \alpha_{1}^{2}+\alpha_{2}^{2}+\alpha_{3}^{2}=1
\end{array}
$$

where the $\alpha_{i}$ are real scalers and the $R_{i}$ and $S_{i}$ are the first and second regular representations respectively of the real quaternions [3].

Similarly, in the case $p=q=2$, Smith [6] shows that any such matrix has exactly one of the following forms:

$$
\begin{array}{lc}
\text { (iii) } \alpha_{1} R_{1} P+\alpha_{2} S_{2} P+\alpha_{3} S_{3} P, & \alpha_{2}^{2}+\alpha_{3}^{2}-\alpha_{1}^{2}=1 \\
\text { (iv) } \alpha_{1} S_{1} P+\alpha_{2} R_{2} P+\alpha_{3} R_{3} P, & \alpha_{1}^{2}-\alpha_{2}^{2}-\alpha_{3}^{2}=-1
\end{array}
$$

where $P=I_{2} \dot{+}\left(-I_{2}\right)$.

A further examination of these papers leads to

Theorem 3: If $\mathrm{Z}$ is a $4 \times 4 \mathrm{P}$-orthogonal, $\mathrm{P}$-skew-symmetric matrix there exists a $4 \times 4$ P-orthogonal, $\mathrm{P}$-skew-symmetric matrix $\mathrm{B}$ such that $\mathrm{ZB}=-\mathrm{BZ}$.

Proof: There are four cases to consider.

Case $1, Z=\alpha_{1} R_{1}+\alpha_{2} R_{2}+\alpha_{3} R_{3}$. If $\alpha_{3} \neq 0$, choose arbitrary $\beta_{1}^{\prime}, \beta_{2}^{\prime}$ and set

$$
\beta_{3}^{\prime}=-\frac{1}{\alpha_{3}}\left(\alpha_{1} \beta_{1}^{\prime}+\alpha_{2} \beta_{2}^{\prime}\right)
$$

Let $x=\beta_{1}^{\prime 2}+\beta_{2}^{\prime 2}+\beta_{3}^{\prime 2}$ and set $\beta_{i}=\frac{\beta_{i}^{\prime}}{\sqrt{x}}, i=1,2,3$. If $\alpha_{3}=0$, let $\beta_{1}=\beta_{2}=0$ and $\beta_{3}=1$. Clearly, in either situation

and

$$
\alpha_{1} \beta_{1}+\alpha_{2} \beta_{2}+\alpha_{3} \beta_{3}=0
$$

$$
\beta_{1}^{2}+\beta_{2}^{2}+\beta_{3}^{2}=1 \text {. }
$$

Letting $B=\beta_{1} R_{1}+\beta_{2} R_{2}+\beta_{3} R_{3}$, by (3) $B$ is $P$-orthogonal, $P$-skew-symmetric and by (2)

$$
Z B=-B Z
$$

Case 2, $Z=\alpha_{1} S_{1}+\alpha_{2} S_{2}+\alpha_{3} S_{3}$. Choose $\beta_{i}, i=1,2,3$ as in Case 1 and let

$$
B=\beta_{1} S_{1}+\beta_{2} S_{2}+\beta_{3} S_{3}
$$

Case 3, $Z=\alpha_{1} R_{1} P+\alpha_{2} S_{2} P+\alpha_{3} S_{3} P$. The matrix $B=\beta_{1} R_{1} P+\beta_{2} S_{2} P+\beta_{3} S_{3} P$ will be $P$-orthogonal, $P$-skew-symmetric if

and $Z B=-B Z$ if

$$
\beta_{2}^{2}+\beta_{3}^{2}-\beta_{!}^{2}=1
$$




$$
\alpha_{2} \beta_{2}+\alpha_{3} \beta_{3}-\alpha_{1} \beta_{1}=0 .
$$

If $\alpha_{1}+\alpha_{2} \neq 0$, set $\beta_{1}=\frac{\alpha_{3}}{\alpha_{1}+\alpha_{2}}, \beta_{2}=\frac{-\alpha_{3}}{\alpha_{1}+\alpha_{2}}$, and $\beta_{3}=1$. Clearly, (4) and (5) are satisfied. If $\alpha_{1}=\alpha_{2}=0$, set $\beta_{1}=\beta_{3}=0, \beta_{2}=1$. Again (4) and (5) are satisfied. If $\alpha_{1}=-\alpha_{2} \neq 0$, since clearly $\alpha_{3}= \pm 1$, set $x=\frac{1}{1+\alpha_{1}^{2}}$ and let $\beta_{1}=0, \beta_{3}=\alpha_{3} \alpha_{1} \sqrt{x}, \beta_{2}=\sqrt{x}$. Again (4) and (5) are satisfied.

Case 4, $Z=\alpha_{1} S_{1} P+\alpha_{2} R_{2} P+\alpha_{3} R_{3} P$. Let $B=\beta_{1} S_{1} P+\beta_{2} R_{2} P+\beta_{3} R_{3} P$ where the $\beta_{i}$ are chosen as in case 3 .

\section{Proof of Theorem 2}

In order to prove Theorem 2 it is convenient to first prove the following lemmas.

LEMmA 1: (i) If $\mathrm{B}$ is $\mathrm{P}$-skew-symmetric then $\mathrm{B}^{\prime}=-\mathrm{PBP}$.

(ii) If $\mathrm{B}$ is $\mathrm{P}$-skew-symmetric and $\mathrm{P}$-orthogonal than $\mathrm{B}^{2}=-\mathrm{I}$.

Lemma 2: If $\mathrm{Z}$ is a P-orthogonal, P-skew-symmetric matrix and $|\mathrm{c}| \leqslant 2$ then

$$
\mathrm{Y}=\frac{\sqrt{4-\mathrm{c}^{2}}}{2} \mathrm{I}+\frac{\mathrm{c}}{2} \mathrm{Z} \text { is P-orthogonal. }
$$

Proof: By direct computation,

$$
\begin{aligned}
Y P Y^{\prime} & =\left(\frac{\sqrt{4-c^{2}}}{2} I+\frac{c}{2} Z\right) P\left(\frac{\sqrt{4-c^{2}}}{2} I+\frac{c}{2} Z^{\prime}\right) \\
& =\frac{4-c^{2}}{4} P+\frac{c^{2}}{4} Z P Z^{\prime}+\frac{\sqrt{4-c^{2}}}{2}\left(Z P+P Z^{\prime}\right) .
\end{aligned}
$$

However, $Z P Z^{\prime}=P$ by (1) and by Lemma 1

$$
Z P+P Z^{\prime}=Z P+P(-P Z P)=Z P-Z P=0 .
$$

Thus $Y P Y^{\prime}=\frac{4-c^{2}}{4} P+\frac{c^{2}}{4} P+O=P$ and by (1) $Y$ is $P$-orthogonal.

Lemma 3: If $\mathrm{Z}$ is $\mathrm{P}$-orthogonal, $\mathrm{P}$-skew-symmetric and $|\mathrm{c}| \leqslant 2$, and if $\mathrm{B}$ is $\mathrm{P}$-orthogonal, $\mathrm{P}$ skew-symmetric such that $\mathrm{ZB}=-\mathrm{BZ}$, then $\mathrm{A}=\left(\frac{\sqrt{4-\mathrm{c}^{2}}}{2} \mathrm{I}+\frac{\mathrm{c}}{2} \mathrm{Z}\right) \mathrm{PB}^{\prime} \mathrm{P}$ satisfies $[\mathrm{A}, \mathrm{B}]=\mathrm{cZ}$.

Proof: $A B=\left(Y P B^{\prime} P\right) B=(Y P)\left(B^{\prime} P B\right)=(Y P) P=Y$

$$
\begin{aligned}
& =\frac{\sqrt{4-c^{2}}}{2} I+\frac{c}{2} Z \\
B A & =B\left(Y P B^{\prime} P\right)=\frac{\sqrt{4-c^{2}}}{2} B P B^{\prime} P+\frac{c}{2} B Z P B^{\prime} P \\
& =\frac{\sqrt{4-c^{2}}}{2} I+\frac{c}{2} B Z P B^{\prime} P \\
& =\frac{\sqrt{4-c^{2}}}{2} I-\frac{c}{2} Z B P B^{\prime} P
\end{aligned}
$$




$$
=\frac{\sqrt{4-c^{2}}}{2} I-\frac{c}{2} Z
$$

Thus $[A, B]=A B-B A=c Z$.

Corollary: The matrix A defined in Lemma 3 is P-orthogonal, P-skew-symmetric.

Proof: By Lemmas 1 and $2 A$ is the product of two $P$-orthogonal matrices. Hence $A$ is $P$ orthogonal. Also

$$
\begin{aligned}
A & =\left(\frac{\sqrt{4-c^{2}}}{2} I+\frac{c}{2} Z\right) P B^{\prime} P \\
& =\frac{\sqrt{4-c^{2}}}{2} P B^{\prime} P+\frac{c}{2} Z P B^{\prime} P .
\end{aligned}
$$

By Lemma $1, \frac{\sqrt{4-c^{2}}}{2} P B^{\prime} P=-\frac{\sqrt{4-c^{2}}}{2} B$ which is $P$-skew-symmetric. Furthermore $\left(\frac{c}{2} Z P B^{\prime} P\right)^{\prime}=\frac{c}{2} P B P Z^{\prime}$

$$
\begin{aligned}
& =-\frac{c}{2} B^{\prime} Z^{\prime} \quad \text { by Lemma } 1 \\
& =\frac{c}{2} Z^{\prime} B^{\prime} \quad \text { since } Z B=-B Z \\
& =-\frac{c}{2} P Z P B^{\prime} \quad \text { by Lemma } 1 \\
& =-\frac{c}{2} P\left(Z P B^{\prime} P\right) P \text {. }
\end{aligned}
$$

Thus $A$ is the sum of two $P$-skew-symmetric matrices and hence $A$ is $P$-skew-symmetric.

In the $4 \times 4$ case, the existence of the matrix $B$ is given by Theorem 3 . Thus Theorem 3 , Lemma 3 , and the Corollary complete the proof of Theorem 2 .

\section{Conclusion}

Theorem 2 provides a converse to the theorems of Pearl [4] and Smith [6]. While Theorem 2 is restricted to the $4 \times 4$ case, the results of section 3 refer to the general $n \times n$ case. Smith [8] has generalized Theorem 1 to the $n \times n$ case. Perhaps the results of section 3 can be applied to find a converse of that result.

\section{References}

[1] Albert, A. A., and Muckenhoupt, B., On matrices of trace zero, Michigan Math. J. Vol. 4, 1-3, (1957).

[2] Katz, L., and Olkin, I., Properties and factorizations of matrices defined by the operation of pseudo-transposition, Duke Math. J. Vol. 20, 331-337, (1953).

[3] MacDuffee, C. C., Orthogonal matrices in four-space, Canadian J. Math. Vol. 1, 69-72, (1949).

[4] Pearl, M., On a Theorem of M. Riesz, J. Res. Nat. Bur. Stand. (U.S.), 62, No. 3, 69-72 (Mar. 1959).

[5] Shoda, K., Einige Satz über Matrizen, Japanese J. Math. Vol. 13, 361-365, (1936).

[6] Smith, J. M., Additional Remarks on a Theorem of M. Riesz, J. Res. Nat. Bur. Stand. (U.S.), 71 B, No. 1, 43-46 (Jan.Mar. 1967).

[7] Smith, J. M., On the Existence of Certain Matrices, Portugaliae Mathematica, Vol. 30, 93-95, (1971).

[8] Smith, J. M., A Theorem on Matrix Commutators, J. Res. Nat. Bur. Stand. (U.S.), 75B, No. 1, 17-21 (Jan.-Mar. 1971). 